\title{
Can BATMAN Replace RPL for loT Applications?
}

\author{
Saba Farooq Abbasi \\ Dept. of Telecommunication \\ Engineering, UET, Taxila, \\ 34295, Pakistan
}

\author{
Adeel Akram \\ Dept. of Telecommunication \\ Engineering, UET, Taxila \\ 34295, Pakistan
}

\author{
Sania Mushtaq \\ Dept. of Telecommunication \\ Engineering, UET Taxila, \\ 34295, Pakistan
}

\author{
Atif Azad \\ Department of Electronics \& Communication Engineering, \\ Technical University Darmstadt, 64289, Germany
}

\begin{abstract}
Routing is one of the most imperious research areas in the Internet of Things (IoT). Routing protocol indicates nodes communication, information dissemination and selection of the best route to reach the destination. This paper presents a comparison between the performance analysis of two routing protocols, RPL and BATMAN, used for Ad-hoc mesh and IoT networks, to observe their strengths and limitations. The analysis is based on evaluation of protocol's packet delivery ratio, packet delay, and routing overhead. Network Simulator 3 (NS-3) is used to simulate the scenarios in order to observe the performance with different number of nodes and varying distance to the destination node. This work will allow the researchers to choose the best suitable protocol for required applications and to have better knowledge of protocols applicability for different IoT scenarios.
\end{abstract}

\section{General Terms}

IoT, routing, Wireless and Mobile Communication.

\section{Keywords}

BATMAN, Internet of Things (IoT), NS-3, routing, RPL.

\section{INTRODUCTION}

To make a society vulnerable of less human intervention, smart entities capable of sending and receiving information in the form of a data over a network are required. This concept is leading a new dimension in the area of communications which is termed as Internet of Things (IoT). For both academia and industry, IoT is the focus of great interest over the last few years. It has applications in smart city, health care, defense, smart buildings, etc. Each application encompasses different scenario with different type of network connectivity e.g. Smart Campus is erected on system convergence, such as Campus Network (wired and wireless network) or Mobile Network [1]. IoT nodes are composed of different modules such as sensors modules, wireless communication modules, power modules, etc. These nodes should be capable to endorse network mobility and take the shortest time to find network routing.

There is no globally accepted standard for IoT routing yet and finding a better suitable routing protocol is of great worth. Over the recent years a momentous effort has been made to guesstimate the performance of IoT routing protocols. [2] discussed some commonly used IoT protocols such as IPv6 over Low-Power Wireless Personal Area Networks
(6LOWPAN), Message Queuing Telemetry Transport (MQTT), Routing Protocol for Low-power and Lossy Networks (RPL) and Constrained Application Protocol (CoAP). Authors also compared protocols for Mobile Ad-Hoc Networks (MANET) and have discussed challenges to these protocols.

Ad-Hoc wireless network is a pool of dynamically and capriciously dispersed mobile nodes which do not have any fixed infrastructure. Great similarities in topological distribution and nodes movement of IoT and Ad-Hoc are observed in [3], authors evaluate the performances of existing Ad-Hoc networks routing protocols, Ad-Hoc On-Demand Vector (AODV), Dynamic Source Routing (DSR) and Optimized Link State Routing (OLSR) to exploit a suitable protocol structure for IoT.

Performance evaluation of Better Approach to Mobile Ad-hoc Network (BATMAN), DSR and OSLR is reviewed [4], by changing the traffic, no. of nodes and node mobility and concluded that BATMAN shows better performance in case of high network mobility.

[5] analyzed the behavior of OSLR and BATMAN in stairs environment, based on MANET testbed, and they concluded that performance of these protocols decreases by increasing the number of nodes and nodes mobility.

[6] made the performance comparison of AODV UU and BATMAND. BATMAND is implementation of BATMAN in a system that is Linux based and implementation of AODV in a Linux-based system is named as AODV Uppsala University (AODV UU). Authors implemented four nodes to evaluate the performance of BATMAND and AODV UU for mobile scenario and found that higher the number of hops and source node movement, greater is the packet lost, low throughput, and high jitter. They determined that BATMAN performed comparatively better in every measured metric except jitter.

[7] observed some of the metrics such as packet overhead, path quality, and connectivity to evaluate performance of RPL but they did not compare RPL with any other routing protocol.

[8] encounter the comparison analysis between RPL and Lightweight On-demand Ad-hoc Distance-vector Routing Protocol (LOADng) using IEEE 802.11 wireless interface and Contiki COOJA simulator to analyze performance metrics like overhead and different traffic patterns. They have 
concluded that RPL is not the best choice for P2P and P2MP traffic.

A Comprehensive study of RPL is carried [9] and when compared with collection tree protocol (CTP) using Contiki/COOJA as the simulation tool, determined that RPL shows better performance in term of energy consumption and high packet reception ratio.

[10] compared RPL and LOADng in home automation scenario with mix type of traffic flow and realistic nonuniform dense network topology. [10] used Contiki/COOJA as a simulation tool. Graphs of their simulated results display that RPL offers shorter delay, lesser overhead and low memory consumption than LOADng in such scenarios.

Both RPL and BATMAN have been compared with many other routing protocols but there has been no direct comparison of RPL and BATMAN as per our best knowledge. The inclusive objective of this simulation study is to analyze the performance of BATMAN and RPL routing protocols in IoT environment and to provide useful information and aid in network design choices.

The paper is ordered as follows: the section I comprises of introduction and related work, in section II overview of the RPL and BATMAN is presented in a brief way, III presents metrics of interest, IV describes simulation setup, results evaluation is covered in section V and section VI concludes the paper and also covers the recommendations.

\subsection{Protocols Overview}

we have considered the routing mechanism of existing routing protocols such as RPL and BATMAN and compared their performance for different IoT scenarios. The mechanism of these routing protocols is as follows:

\subsubsection{Routing Protocol for Low Power and Lossy Networks (RPL)}

RPL is proactive distance vector routing protocol for the IPv6 founded Lossy Networks [11] and was standardized by the IETF in 2011. It does not depend on any explicit features of a link-layer technology. Link layer can be Power Line Communication (PLC) using IEEE 802.15.4, Bluetooth or low power Wi-Fi [12]. The concept of RPL is Directed acyclic graph which is a tree-like structure to specify the route to the root node [13]. A network may consist of more than one DODAG that conglomerate to form RPL instance. There are three important RPL control message to construct RPL DODAG. DODAG start to construct when the root node is switched on and it get-go broadcasting destination object information (DIO) message in the downward direction. In the case when no announcement is heard and a new node wants to join the DODAG then node broadcast DODAG information solicitation (DIS) message. DIS is basically a call "Is there any DODAG". When the node receives DIO message from the root or any other node, it sends DODAG advertisement object (DAO) message. DAO is a request from a child to the parent node or root to join DODAG. When node receives DIO it stores the address of transmitter in the list of its possible parents. A node may have more than one parent, preferable parent (selected on the base of the objective function and has the smallest rank in its list) is then used to route the packet to the root and keeps the other parent as a backup. Each node announces and triggered by a timer. Timer's interval upsurges exponentially. I_min is the tiniest possible interlude between two DIOs and I_double symbolizes number of times I_min is doubled afore getting to a constant value, so the maximum interval amid the two DIOs is expressed in equation (1) [7].

$$
I_{\max }=I_{\min } * 2 I_{\text {doubling }}
$$

The total time that a packet takes to reach the destination auspiciously is known as round trip time (RTT) discussed in [14]. Fig. 1. shows DODAG building process.

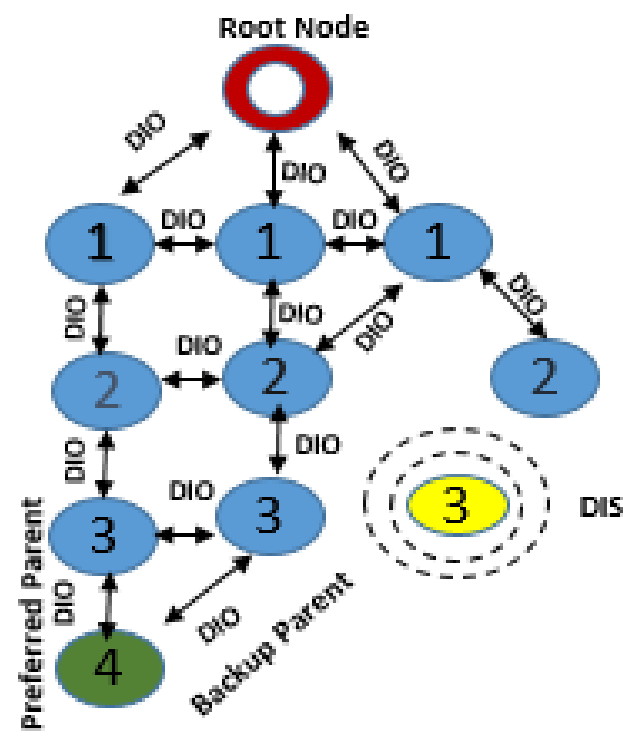

Figure 1: RPL DODAG Construction

DODAG ID has 128 bits IPV6 address. This ID is given to the root only, other sensing nodes are connected to this border router and together they all form sort of island where they share the common prefix. Border router got IP address and has a prefix in IP address and this prefix is shared by every node in the island.

\begin{tabular}{|c|c|c|}
\hline RPL instance ID & Version Number & Rank \\
\hline 1byte & 1byte & 2byte \\
\hline \multicolumn{3}{|c|}{ DODAG ID } \\
\hline \multicolumn{2}{|c|}{$\mathbf{1 2 8}$ bits } \\
\hline
\end{tabular}

Figure 2: RPL message structure

RPL generally offers two modes of operation for the communication, storing mode and non-storing mode [15]. Loss of traffic control in routing topology may cause the formation of a loop which will lead to the failure of routing link. RPL yield loop avoidance mechanism based on the nodes ranks. It also has the mechanism to repair the faulty link [16].

\subsubsection{Better Approach to Mobile Ad-Hoc Network (BATMAN)}

BATMAN is proactive routing protocol [4] for Ad- Hoc and wireless networks. BATMAN functions at network layer as well as at data link layer with kernel-space implementation. Workings principle of BATMAN is quite simple [17, 18], unlike OSLR [19] where the whole routing path is calculated by each node in the network. Each node in BATMAN network perceives and maintains the information only about 
the best next-hop towards all other nodes, also known as linklocal neighbor.

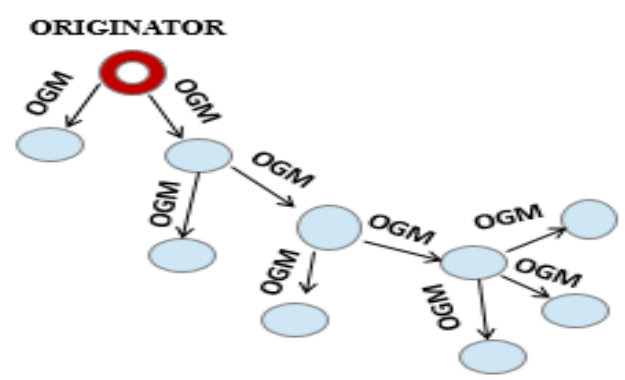

Fig 3: BATMAN packet flow through a network

BATMAN has a hello message also known as Originator message or OGM, having packet size of 52 bytes including IP and UDP overhead and is broadcasted by every node. OGM typically contains sending node address, originator address, a unique sequence number and packet's time to live (TTL). On receiving OGM the neighboring node retransmits OGM to its own local-link neighbors and so on. This process continues until every node in the network receives OGM at least ones or their time to live (TTL) gets expired. Originator adds a sequence number to every OGM it generates and every time a new OGM is generated this sequence number is increased. This sequence number is used by the routing algorithm to pick best next-hop. In BATMAN version IV Transmit Link Quality (TQ), previous sender and HNA length fields are added to overcome shortcoming such as network overhead and link quality

In order to prevent routing loop and traffic overflow, a node on receiving an existing OGM, drops it. Flag bits are used to identify if the OGM being received by any node is from a link-local (direct) neighbor or not. Total number of OGM messages sent over every interval is $\mathrm{N}^{2}$ [20], where $\mathrm{N}$ is the total number of nodes in any network.

\begin{tabular}{|l|c|c|c|}
\hline Version & Flags & TTL & Gateway Flags \\
\hline \multicolumn{2}{|c|}{ Sequence Number } & Gateway Port \\
\hline \multicolumn{3}{|c|}{ Originator Address } \\
\hline \multicolumn{3}{|c|}{ Precedent Sender Address } \\
\hline & HNA Length & TQ \\
\hline
\end{tabular}

Figure 4: BATMAN IV OGM format

Table 1. Properties comparison of BATMAN and RPL

\begin{tabular}{|l|l|l|}
\hline $\begin{array}{l}\text { PROTOCOL's } \\
\text { PROPERTY }\end{array}$ & BATMAN & RPL \\
\hline IP Version & IPV4,IPV6 & IPV6 \\
\hline Routing Layer & Layer 2 Routing & Layer3 Routing \\
\hline Reactive & No & No \\
\hline Proactive & Yes & Yes \\
\hline Loop Free & No & No \\
\hline
\end{tabular}

\section{METRICS OF INTEREST}

In order to evaluate the performance of routing protocols, three different quantitative metrics are used. This work evaluates the performance of selected protocols by considering the following parameters.

\subsection{Packet Delivery Ratio}

PDR is the ratio between total number of packets sent by the source and number of packets that are received by the destination node. Higher the valve of PDR, better will be the performance of routing protocol. Packet delivery ration can be calculated as

$$
P D R=\frac{P_{R}}{P_{t}}
$$

Where $P_{t}$ is use for total packet sent by application layer and $\mathrm{P} \square_{\mathrm{R}}$ represents the received packets by the destination node.

\subsection{Routing Overhead}

The overall packets transmitted throughout the whole simulation period is labeled as routing overhead. Additional transmissions used to route the data packet to destination e.g., hello message, acknowledgement, any control message etc. cause network overhead. These are fundamentally extra bytes transmit during network simulation.

\subsection{End-to-End Delay}

How long does a packet take to reach from its source to the destination is measured at MAC layer and called end-to-end delay. It indicates competency of protocol to take the best route to the destination.

\section{SIMULATION SETUP}

$[21,22]$ have implemented BATMAN and RPL in NS-3 respectively. Reported work uses their basic implementation to compare different routing metrics of BATMAN and RPL using Network Simulator 3 (NS-3) as a simulation tool. NS-3 is a new, promising, trustworthy open source discrete event simulator, fully equipped with IPv6 Supportive stack model, along with a trustful WPAN model with 6LoWPAN adaptation function. RPL DAOs is not included in simulations, in order to reduce the network complexity as discussed in [22]. Simulations are performed using a different number of nodes (10 to 50) and the distance of these sensor nodes is also varying form their destination node. Nodes are moving randomly in a rectangular flat space of fixed area. The overall simulation period for each simulation setup is set to 300 seconds. Nodes generate a constant bit rate traffic using 802.11 wireless interface. Use of 802.11 wireless interface is generally not recommended for LLN but we can still use it as [8] explained this.

Table 2. Simulation parameters for different simulations

\begin{tabular}{|l|l|}
\multicolumn{2}{c}{ run } \\
\hline Simulation Platform & NS-3 \\
\hline No. of nodes & $10-50$ \\
\hline Data packet size & 64 bytes \\
\hline Wireless MAC Protocol & $802.11 \mathrm{~b}$ \\
\hline Loss model & Friis propagation loss model \\
\hline Simulation time & $300 \mathrm{~s}$ \\
\hline Node velocity & $0-20 \mathrm{~m} / \mathrm{s}$ \\
\hline
\end{tabular}




\section{SIMULATION RESULTS AND DISCUSSION}

Figure 5 illustrates average end-to-end delay versus node density curve for both protocols. Both RPL and BATMAN showed an increase in delay as the number of node increases however, it is observed that BATMAN has the lower end-toend delay as compared to RPL at every node density. When node density is increased delay performance of BATMAN competes with that of RPL and BATMAN manages to maintain a relatively low packet delay and show better performance than RPL. This is due to the fact that OGM notion of BATMAN speeds the best root selection process whereas, RPL DODAG construction is a time-consuming practice thus causes more delay in the network.

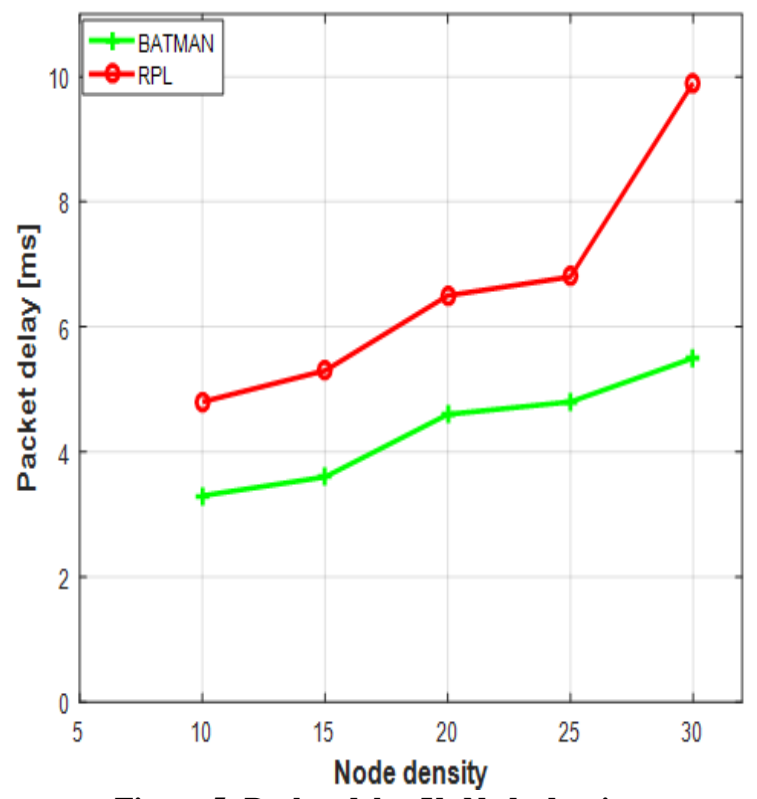

Figure 5: Packet delay Vs Node density

Packet delivery ration with varying distance to the destination, with 50 nodes, is shown in figure 6. PDR and distance to the destination node have an inverse relation, which is evident from the figure. Poor packet delivery rate may have caused by many reasons, such as intrusions, collisions and signal weakening. RPL shows a poor PDR performance than BATMAN, as the distance from the destination node is increased. The mobile nodes involvement scenarios may cause the sensor nodes to move out of the range of their parent node. When the nodes move away from their parent node they may lose their connectivity with them and have to choose a new preferred parent on their way to the root node, so the whole DODAG building process takes place all over again causing fewer packets to move to the root node for a particular simulation run.

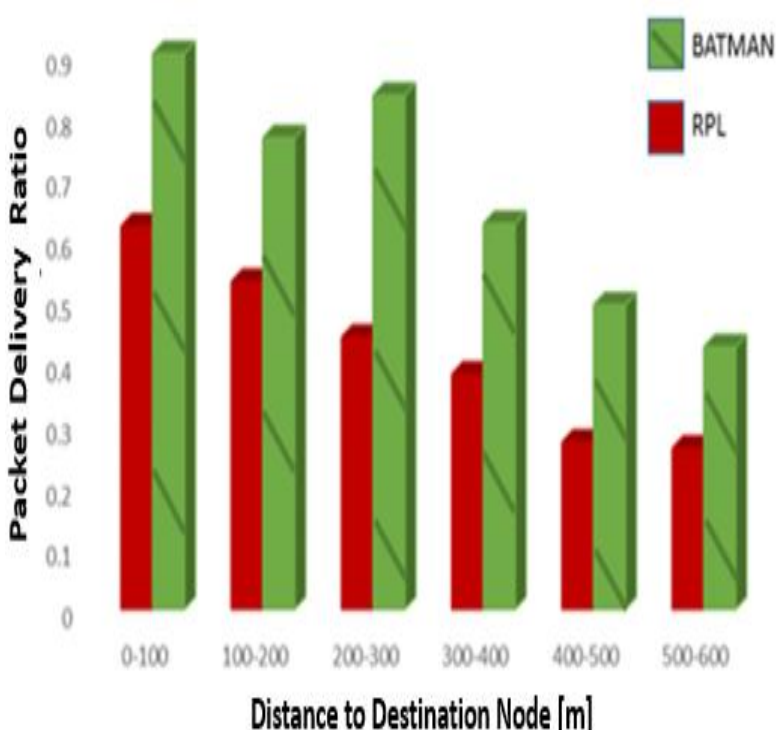

Figure 6: Packet delivery ratio versus distance to the sink

Figure 7 displays that RPL routing overhead is mainly caused by control packets (DIO, DIS) and NS3 Wi-Fi module. Control messages play a vital role in the construction of DODAG resulting in increased routing overhead, which will be reduced as soon as the DODAG construction process is completed. Thus, most of the RPL overhead appears at the beginning of the simulation. Overhead of BATMAN is comparatively higher than RPL because every node in a BATMAN network continues to broadcast OGMs packet at equal intervals, for the entire simulation time period. These OGMs occupies major portion in overall network overhead.

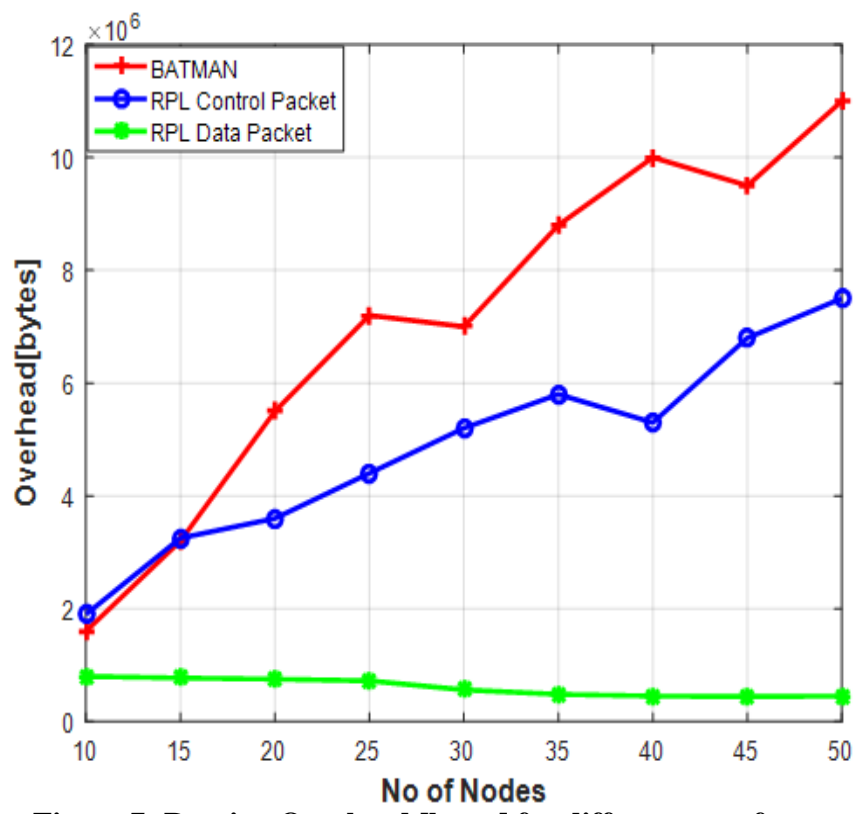

Figure 7: Routing Overhead [bytes] for different no. of nodes

\section{CONCLUSION AND RECOMMENDATIONS}

The reported work has evaluated RPL and BATMAN routing protocols for mobile network scenarios. The comparative analysis is done using three performance metrics i.e. routing 
overhead, average end to end delay and packet delivery ratio, at varying nodes density $(10-50)$ and distance to the root node $(100-600 \mathrm{~m})$. The analysis reveals that BATMAN is a better performer as compared to RPL in terms of packet delivery ratio and average end-to-end delay for dense networks and also when the factor of mobility is considered. When it comes to network overhead BATMAN lags in performance than RPL.

For routing purposes in IoT, RPL is used frequently. RPL uses IPV6 addresses, whereas IPV4 is commonly in practice in already deployed networks. While BATMAN is layer 2 routing protocol which can be implemented using IPv4, IPv6, IPX, DHCP. Performance of RPL decreases in case of highly mobile networks. [23] reported some other limitations of RPL.

In light of the proposed comparative analysis, BATMAN finds a viable choice and potential competitor to replace RPL protocol. In order to make the statement strong simulations have been done which depicts that RPL poses more delay and less packet delivery ratio, which can cause hinders in the idea of real-time IoT scenario. One of the important parameters to measure the performance of the IoT environment is power consumption. BATMAN protocol consumes more power than RPL due to its overhead, so if the network is power constrained it is better to choose RPL over BATMAN, otherwise BATMAN is more suitable choice when overall network performance is required.

\section{REFERENCES}

[1] Guo, M. and Zhang, Y., 2015 The research of smart campus based on Internet of Things \& cloud computing," Wireless communication, Networing and Mobile Computing (WiCOM 2015), $11^{\text {th }}$ Inter- national Conference, pp. 1-6. IET, 2015.

[2] Noorul, T.M., Pramila, R.S. and Islam, N., 2017 An analysis of routing protocols in manets and Internet of things. In IoT and Application (ICIOT), IEEE International Conference on (pp. 1-8).

[3] Xin, H.M. and Yang, K., 2015, April. Routing protocols analysis for Internet of Things. In Information Science and Control Engineering (ICISCE), IEEE 2nd International Conference on (pp. 447-450)

[4] Sandhu, D.S. and Sharma, S., 2012. Performance evaluation of batman, DSR, OLSR routing protocols-A review. Journal of Information and Operations Management, 3(1), p.225.

[5] Kulla, E., Hiyama, M., Ikeda, M. and Barolli, L., 2012. Performance comparison of OLSR and BATMAN routing protocols by a MANET testbed in stairs environment. Computers \& Mathematics with Applications, 63(2), pp.339-349.

[6] Prathama, M.P. and Hertiana, S.N., 2013, October. An analysis comparison of AODV UU and batmand performance for mobile ad-hoc network. In Information Technology and Electrical Engineering (ICITEE), IEEE International Conference on (pp. 260-265).

[7] Tripathi, J., de Oliveira, J.C. and Vasseur, J.P., 2010, March. A performance evaluation study of rpl: Routing protocol for low power and lossy networks. In Information Sciences and Systems (CISS), IEEE 44th Annual Conference on (pp. 1-6).
[8] Yi, J., Clausen, T. and Igarashi, Y., 2013, December. Evaluation of routing protocol for low power and Lossy Networks: LOADng and RPL. In Wireless Sensor (ICWISE), 2013 IEEE Conference on (pp. 19-24).

[9] Long, N.T., De Caro, N., Colitti, W., Touhafi, A. and Steenhaut, K., 2012, November. Comparative performance study of RPL in wireless sensor networks. In Communications and Vehicular Technology in the Benelux (SCVT), 2012 IEEE 19th Symposium on (pp. 1$6)$

[10] Vučinić, M., Tourancheau, B. and Duda, A., 2013, April. Performance comparison of the RPL and LOADng routing protocols in a home automation scenario. In Wireless Communications and Networking Conference (WCNC), 2013 IEEE (pp. 1974-1979).

[11] Iova, O., Theoleyre, F., Watteyne, T. and Noel, T., 2017. The Love-Hate Relationship between IEEE 802.15. 4 and RPL. IEEE Communications Magazine, 55(1), pp.188-194.

[12] Salman, T. and Jain, R., 2015. Networking Protocols and Standards for Internet of Things. Internet of Things and Data Analytics Handbook (2015), pp.215-238.

[13] Gaddour, O. and Koubâa, A., 2012. RPL in a nutshell: A survey. Computer Networks, 56(14), pp.3163-3178.

[14] Adya, A., Bahl, P., Padhye, J., Wolman, A. and Zhou, L., 2004, October. A multi-radio unification protocol for IEEE 802.11 wireless networks. In Broadband Networks, 2004. BroadNets 2004. Proceedings. First International Conference on (pp. 344-354).

[15] Ko, J., Jeong, J., Park, J., Jun, J.A., Gnawali, O. and Paek, J., 2015. DualMOP-RPL: Supporting multiple modes of downward routing in a single RPL network. ACM Transactions on Sensor Networks (TOSN), 11(2), p.39.

[16] Oliveira, A. and Vazão, T., 2016. Low-power and lossy networks under mobility: A survey. Computer Networks, 107, pp.339-352.

[17] Kulla, E., Ikeda, M., Barolli, L. and Miho, R., 2010, November. Impact of source and destination movement on MANET performance considering BATMAN and AODV protocols. In Broadband, Wireless Computing, Communication and Applications (BWCCA), 2010 International Conference on (pp. 94-101).

[18] Barolli, L., Ikeda, M., De Marco, G., Durresi, A. and Xhafa, F., 2009, May. Performance analysis of OLSR and BATMAN protocols considering link quality parameter. In Advanced Information Networking and Applications, 2009. AINA'09. IEEE International Conference on (pp. 307-314).

[19] Kulla, E., Ikeda, M., Barolli, L., Miho, R. and Kolici, V., 2010, September. Effects of source and destination movement on MANET performance considering OLSR and AODV protocols. In Network-Based Information Systems (NBiS), IEEE 13th International Conference on (pp. 510-515).

[20] Furlan, D., 2011. Analysis of the overhead of BATMAN routing protocol in regular torus topologies. University of Trento, Italy, Tech. Rep.

[21] Bowitz, A.G., Graarud, E.G., Brown, L. and Jaatun, M.G., 2011, September. BatCave: Adding security to the 
BATMAN protocol. In Digital Information Management (ICDIM), 2011 IEEE Sixth International Conference on (pp. 199-204).

[22] Chen, Y.B., Hou, K.M., Chanet, J.P. and El Gholami, K., 2013, September. A RPL based Adaptive and Scalable Data-collection Protocol module for NS-3 simulation platform. In NICST 2103 New Information
Communication Science and Technology for Sustainable Development: France-China International Workshop (pp. 8-p).

[23] Parasuram, A., Culler, D. and Katz, R., 2016. An analysis of the RPL routing standard for low power and lossy networks (Doctoral dissertation, MS thesis, EECS Dept., Univ. California at Berkeley, Berkeley, CA, USA). 